\title{
Objectively-Measured Smartphone Usage, Sleep Quality, and Physical Activity Among Chinese Adolescents and Young Adults
}

Paul H Lee ( $\sim$ paul.h.lee@polyu.edu.hk)

Hong Kong Polytechnic University https://orcid.org/0000-0002-5729-6450

Andy C. Y. Tse

Education University of Hong Kong

Cynthia S. T. Wu

Hong Kong Polytechnic University

Yim Wah Mak

Hong Kong Polytechnic University

Uichin Lee

Korea Advanced Institute of Science and Technology

\section{Research}

Keywords: exercise, gaming, screen, smart device, smartphone monitoring, youth

Posted Date: May 21st, 2020

DOI: https://doi.org/10.21203/rs.3.rs-30312/v1

License: @) (i) This work is licensed under a Creative Commons Attribution 4.0 International License. Read Full License 


\section{Abstract}

Objectives: We studied the association between objectively-measured smartphone usage and objectively-measured sleep quality and physical activity for seven consecutive days among Hong Kong adolescents and young adults aged $11-25(\mathrm{n}=357,67 \%$ female).

Methods: We installed an app that tracked the subjects' smartphone usage and had them wear an ActiGraph GT3X accelerometer on their wrist to measure their sleep quality and physical activity level. Smartphone usage data were successfully obtained from 187 participants (52.4\%).

Results: The participants on average spent 2 hours 46 minutes per day on their smartphone. Multilevel regression showed that, among secondary school students, one minute of daytime smartphone usage was associated with 0.12 minute decrease in total sleeping time that night ( $p=0.042,95 \% \mathrm{Cl}:-0.23,-0.007)$. One minute of bedtime smartphone usage was associated with 0.32 minute increase in wake after sleep onset that night $(p=0.04,95 \% \mathrm{Cl}: 0.02,0.62)$. One minute of smartphone usage during sleep was associated with sleep efficiency $(\beta=0.013 \%, p=0.01,95 \% \mathrm{Cl}$ : $0.003 \%$, $0.023 \%)$ and WASO ( $\beta=-0.05, p=0.04,95 \% \mathrm{Cl}:-0.10,-0.005)$. One minute of daytime smartphone usage was associated with 7.15 steps increase in the number of steps ( $p=0.02,95 \% \mathrm{Cl}: 1.02,13.28)$ among secondary school students and 3.52 steps increase in the number of steps $(p=0.03,95 \% \mathrm{Cl}: 0.37,6.66)$ among university students on the next day.

Conclusion: Time spent on smartphone was associated with total sleeping time, the number of steps, and MVPA among Hong Kong adolescents and young adults.

\section{Introduction}

The use of smartphones has been increasing rapidly in recent years. The number of smartphone users worldwide was 1.5 billion in 2014 and this figure is expected to be doubled in 2020 [1]. Smartphones have substituted for mp3 music players, video players, and handheld game consoles, as they allow easy access to all of these functions at any time and any place at the user's convenience [2]. Smartphones also allow for the taking of instant photos and videos and the sharing of them through the internet. Moreover, smartphones allow users to download attractive and powerful applications for education, entertainment, and stock transactions. Smartphones are becoming so important that $29 \%$ of their owners "can't imagine living without" them [3].

Smartphone ownership and usage are a global phenomenon, with Hong Kong being one of the cities with the highest penetration rates around the world (ranked 8th out of 47 countries or places in 2013 [4]). Local studies have revealed that almost all adults, more than $50 \%$ of primary school students, and more than $90 \%$ of secondary school students possess at least one smartphones [5]. Another study conducted by the Hong Kong Polytechnic University showed that $30 \%$ of school students used smart devices (including smartphones and tablets) for one to four hours per day [6].

While appropriately using smartphones can improve our quality of life, excessive usage is likely to lead to adverse health outcomes. The excessive usage of smartphones was associated with sedentary lifestyles [7], poor sleeping habits [8], and increased waking-time tiredness [8]. A recent large-scale cross-sectional study among adolescents in Norway showed that both the daytime and bedtime use of electronic devices was associated with sleep problems [9], confirming the theoretical pathway that the lighted screen of smartphone suppresses melatonin and affects sleep. Furthermore, increased time spent in bedtime smartphone usage will shorten the user's total sleeping time and time spent in physical activity. 
Note that all of the aforementioned studies suffered a common major limitation, namely the use of the self-report level of smartphone usage, in which the reporting was subjected to recall bias [9], unknown validity and reliability [9], and an inability to track the frequently-changing smartphone usage habit [10].

Objectively-measured data on smartphone usage can be obtained by directly monitoring smartphone activities. Many smartphone monitoring applications have been written, most of which have been aimed at collecting usage information on data transfer and battery power consumption for further hardware development and improvement [1116]. Very few studies have correlated objectively-measured smartphone usage with other outcomes; to the best of our knowledge, there exists only one study correlating objectively-measured smartphone usage with personality traits [17]. To date, there have been no studies on the association between objectively-measured smartphone usage and health outcomes. Here, we studied the association between objectively-measured smartphone usage and objectivelymeasured sleep quality and physical activity (using a wrist-worn accelerometer) measured for seven consecutive days in Hong Kong Chinese adolescents and young adults.

\section{Methods}

\section{Participants}

This study was conducted from March 2017 to May 2018. The participants were recruited from one secondary school (Tai Po Sam Yuk Secondary School, which corresponds to Years 7 to 12 in the US education system) and two universities (Hong Kong Polytechnic University and Education University of Hong Kong) in Hong Kong. In the secondary school, invitation letters were sent to the parents of all students. In the two universities, invitation emails were sent to all undergraduate students. Only those aged 11-25, who were able to speak and read Chinese, and owned a smartphone with the Android operating system were recruited to take part in this study. Phones with the Apple iOS operating system were excluded because Apple iOS app programmers are not allowed to collect smartphone usage data continuously. Written consent was obtained from all of the participants. For participants under the age of 18, parental written consent was also obtained. As a token of appreciation, a supermarket cash coupon worth HK \$100 (approximately US \$13) was given to the participants after the data were collected. This study was approved by the Human Subjects Ethics Sub-Committee of the Hong Kong Polytechnic University.

\section{Data collection}

Objectively-measured smartphone usage. We installed a smartphone usage tracking app (created by our team) on the smartphone of all of the participants' smartphone for seven consecutive days. This app tracked the opening and closing of all apps in the smartphone. No private or personal data, such as the contents of instant messages and the web pages that were browsed, were collected. For those participants who possessed more than one smartphone, we installed the app in their most commonly-used phone. We defined an app as being used when it was running in the graphical interface. It should be noted that the opening of one app would trigger the closure of the app currently being used, if any. The usage time was tracked by seconds. The names of the apps being used, their opening time, and closing time were stored in the smartphone and uploaded to a remote server (Firebase) once internet connection was available, and all uploaded data were removed from the smartphone.

Sleep quality and physical activity level. During the seven-day monitoring period, all of the participants concurrently wore an ActiGraph GT3X accelerometer. This is a wrist-worn, water-proof accelerometer that has been validated for measuring sitting time [18], physical activity [19], and sleeping time [20,21]. The participants were instructed to wear the accelerometer on their non-dominant hand for 24 hours a day over seven consecutive days. They were asked to record the removal time in a logbook, if any (for example, when participating in a soccer or basketball competition 
during which the accelerometer might become damaged). Data were collected in 1-minute epoch. To synchronize the accelerometer data with smartphone usage data, when initializing the accelerometers Greenwich Mean Time +8 , obtained via the internet, were used.

\section{Data processing}

Sleep quality and physical activity level. Peak acceleration in the three axes was extracted from the raw acceleration. The resultant acceleration of the raw acceleration from three axes was summarized by counts per minute (CPM). CPM of $\geq 4,514$ of the vertical axis was classified as moderate-to-vigorous-intensity activity (MVPA) [22]. Non-wearing time was defined as consecutive zero counts for 60 or more minutes. The amount of wearing time in a day was computed by subtracting the non-wearing time and sleeping time (determined by the accelerometer) from 24 hours. A valid day must include at least 10 hours of wearing time excluding sleeping time, and only those who provided a minimum of one valid day were included in the analysis. All of the minutes recorded from the accelerometers were classified as either sleep or awake using Sadeh's algorithm [23]. According to Sadeh's algorithm, the sleep index of a minute is defined as $7.601-(0.065 \times$ AVG $)-(1.08 \times$ NATS $)-(0.056 \times S D)-(0.703 \times L G)$, where AVG is the average CPM of the 11 minutes centered at the current minute, NATS is the number of minute with CPM between 50 to 99 at the 11-min period, SD is the standard deviation of the current and five preceding minutes, and LG is the natural log of the CPM +1 (to avoid log of 0 ) at the current minute. Recorded minutes with sleep index of $\geq-4$ will be classified as sleep, and awake otherwise. Sleep onset was defined as the first 15 minutes of consecutive sleeping minutes, and awakening time was defined as the first 15 minutes of consecutive wake minutes. The total sleeping period was measured as beginning at sleep onset and ending at awakening. Total sleeping time was defined as the sleeping time within the total sleeping period. Sleep efficiency was defined as the total sleeping time divided by the total sleeping period. Wake after sleep onset (WASO) was defined as the waking time within the total sleeping period.

Objectively-measured smartphone usage. All recorded smartphone applications were categorized under social network (Facebook, Twitter, Instagram, Weibo, etc.), instant messaging (Whatsapp, Skype, Line, Wechat, etc.), web browsing (including browser and apps developed for browsing specific websites), games and comics (except games plug-ins and guides), multimedia (related to music, video, and image/photo, for example YouTube and MOOV), camera (including beauty-themed photo and video apps such as Meitu), and health (pedometer, GPS distance and speed tracker, etc.). The time spent on each usage session was computed as the difference between the closing time and opening time. Usage sessions of less than 1 second were discarded as such short times were likely to be an indication that the app had been accidentally pressed. All smartphone usage time were classified as either occurring in the daytime (defined as the time from waking up to 1 hour before sleep, where the sleeping time was identified using the accelerometer data), bedtime (defined as 1 hour before sleep), or during sleep. Smartphone usage time during sleep was excluded as the phone usage may not be performed by the smartphone owner.

\section{Statistical analysis}

Descriptive statistics (mean, SD, frequency) were used to summarize the pattern of smartphone usage, sleep quality, and physical activity level. Multilevel regression was used to examine the temporal association between daytime and bedtime smartphone usage with sleep quality that night and physical activity on the following day, adjusted for the data source (secondary school / university), sex, and within-subject correlation. The data from the first day that the smartphone usage tracking app was installed and the accelerometer was distributed were excluded due to the incompleteness of the data. All data analysis was stratified by school type (secondary school versus university) $p$ value of $<0.05$ was considered significant.

\section{Results}


A total of 393 students (156 from the secondary school and 237 from the two universities) agreed to participate, and 357 of them who provided at least one valid day of accelerometer data were included in the current analysis. Table 1 shows the characteristics of the participants. Nearly two-thirds of them were university students. The secondary school sample was balanced in gender, but in the university sample less than one-fourth of them were male. The sleep quality and physical activity level of the two samples were comparable.

Table 1

Demographic characteristics and accelerometer-measured sleep quality and physical activity level of the participants $(n=357)$

\begin{tabular}{|c|c|c|c|}
\hline & $\begin{array}{l}\text { Secondary school } \\
\text { students } \\
\text { (number of participants = } \\
\text { 125) }\end{array}$ & $\begin{array}{l}\text { University students } \\
\text { (number of participants = } \\
232 \text { ) }\end{array}$ & $\begin{array}{l}\text { Overall } \\
\text { (number of participants = } \\
187, \text { number of days = } \\
1,106 \text { ) }\end{array}$ \\
\hline Variable & Frequency (\%) & Frequency (\%) & Frequency (\%) \\
\hline \multicolumn{4}{|l|}{ Sex } \\
\hline Male & $62(50.4 \%)$ & $55(23.7 \%)$ & $117(33.0 \%)$ \\
\hline \multirow[t]{2}{*}{ Female } & $61(49.6 \%)$ & $177(76.3 \%)$ & $238(67.0 \%)$ \\
\hline & Mean (SD) & Mean (SD) & Mean (SD) \\
\hline Age & $18.4(3.3)$ & $18.4(3.3)$ & $18.4(3.3)$ \\
\hline Sleep quality & $\begin{array}{l}\text { Secondary school } \\
\text { students } \\
\text { (number of participants = } \\
32, \text { number of days }=176 \text { ) }\end{array}$ & $\begin{array}{l}\text { University students } \\
\text { (number of participants }= \\
155, \text { number of days }=930 \text { ) }\end{array}$ & $\begin{array}{l}\text { Secondary school students } \\
\text { (number of participants = } \\
187, \text { number of days = } \\
1,106)\end{array}$ \\
\hline $\begin{array}{l}\text { Total sleeping time } \\
\text { (hr) }\end{array}$ & $7.5(1.8)$ & $7.5(1.8)$ & $7.5(1.8)$ \\
\hline Sleep efficiency (\%) & $94.3(4.5)$ & $94.9(4.1)$ & $94.7(4.2)$ \\
\hline $\begin{array}{l}\text { Wake after sleep } \\
\text { onset (min) }\end{array}$ & $25.2(19.9)$ & $22.6(17.8)$ & $23.4(18.5)$ \\
\hline Physical activity level & $\begin{array}{l}\text { Secondary school } \\
\text { students } \\
\text { (number of participants = } \\
123 \text {, number of days = } \\
459 \text { ) }\end{array}$ & $\begin{array}{l}\text { University students } \\
\text { (number of participants = } \\
229, \text { number of days = } \\
1,058 \text { ) }\end{array}$ & $\begin{array}{l}\text { Secondary school students } \\
\text { (number of participants = } \\
352 \text {, number of days = } \\
1,517 \text { ) }\end{array}$ \\
\hline Number of step & $10,315(4,950)$ & $10,003(4,186)$ & $10,095(4,423)$ \\
\hline $\begin{array}{l}\text { Moderate-to-vigorous } \\
\text { physical activity } \\
\text { (min) }\end{array}$ & $162.7(102.6)$ & $137.2(73.2)$ & $144.6(83.6)$ \\
\hline
\end{tabular}

Of the 357 participants, smartphone usage data were successfully tracked for 187 (52.4\%). The smartphone tracking app was written for Android 7.0 or above, and the tracking app did not work with some smartphones installed with earlier Android versions. The daytime and bedtime smartphone usage data are summarized in Table 2. The participants spent nearly 3 hours per day on their smartphone. Secondary school students on average spent 40 more minutes per day on smartphones than university students. The most common type of usage among secondary school students and university students were games and comics apps (1 hour 30 minutes per day) and instant messaging 
apps (1 hour 13 minutes per day), respectively. Bedtime smartphone usage was recorded in 464 (42.0\%) out of the 1,106 measured nights. On average the participants spent 10 minutes per day on their smartphone during bedtime. Smartphone usage during sleep was recorded in 563 (50.9\%) out of the 1,106 measured nights. On average the participants spent 29 minutes per day on their smartphone during sleep.

Table 2

Smartphone usage (min) of the participants (number of participants $=187$, number of days $=1,106$ )

\begin{tabular}{|c|c|c|c|c|c|c|c|c|c|}
\hline \multirow[b]{2}{*}{$\begin{array}{l}\text { Smartphone } \\
\text { usage }\end{array}$} & \multicolumn{3}{|c|}{$\begin{array}{l}\text { Secondary school students } \\
\text { (number of participants = } 32 \text {, } \\
\text { number of days }=176 \text { ) }\end{array}$} & \multicolumn{3}{|c|}{$\begin{array}{l}\text { University students } \\
\text { (number of participants = 155, } \\
\text { number of days }=930 \text { ) }\end{array}$} & \multicolumn{3}{|c|}{$\begin{array}{l}\text { Overall } \\
\text { (number of participants = 187, } \\
\text { number of days }=1,106 \text { ) }\end{array}$} \\
\hline & $\begin{array}{l}\text { Daytime } \\
\text { (mean } \\
(\mathrm{SD}))\end{array}$ & $\begin{array}{l}\text { Bedtime } \\
\text { (mean } \\
(\mathrm{SD}))\end{array}$ & $\begin{array}{l}\text { During } \\
\text { sleep } \\
\text { (mean } \\
(\mathrm{SD}) \text { ) }\end{array}$ & $\begin{array}{l}\text { Daytime } \\
\text { (mean } \\
(\mathrm{SD}))\end{array}$ & $\begin{array}{l}\text { Bedtime } \\
\text { (mean } \\
(\mathrm{SD}))\end{array}$ & $\begin{array}{l}\text { During } \\
\text { sleep } \\
\text { (mean } \\
(\mathrm{SD}))\end{array}$ & $\begin{array}{l}\text { Daytime } \\
\text { (mean } \\
(\mathrm{SD}))\end{array}$ & $\begin{array}{l}\text { Bedtime } \\
\text { (mean } \\
(S D))\end{array}$ & $\begin{array}{l}\text { During } \\
\text { sleep } \\
\text { (mean } \\
(S D))\end{array}$ \\
\hline $\begin{array}{l}\text { Social } \\
\text { network }\end{array}$ & $\begin{array}{l}23.1 \\
(101.9)\end{array}$ & $\begin{array}{l}1.1 \\
(7.0)\end{array}$ & $\begin{array}{l}2.2 \\
(13.8)\end{array}$ & $\begin{array}{l}24.2 \\
(52.1)\end{array}$ & $\begin{array}{l}2.0 \\
(8.8)\end{array}$ & $\begin{array}{l}4.4 \\
(26.8)\end{array}$ & $\begin{array}{l}24.0 \\
(62.7)\end{array}$ & $\begin{array}{l}1.9 \\
(3.8)\end{array}$ & $\begin{array}{l}4.0 \\
(25.2)\end{array}$ \\
\hline $\begin{array}{l}\text { Instant } \\
\text { messaging }\end{array}$ & $\begin{array}{l}54.8 \\
(96.5)\end{array}$ & $\begin{array}{l}3.3 \\
(12.2)\end{array}$ & $\begin{array}{l}9.6 \\
(43.9)\end{array}$ & $\begin{array}{l}73.4 \\
(112.6)\end{array}$ & $\begin{array}{l}3.9 \\
(12.3)\end{array}$ & $\begin{array}{l}11.5 \\
(73.8)\end{array}$ & $\begin{array}{l}70.5 \\
(110.3)\end{array}$ & $\begin{array}{l}3.8 \\
(12.3)\end{array}$ & $\begin{array}{l}11.2 \\
(69.9)\end{array}$ \\
\hline Tools & $\begin{array}{l}16.1 \\
(45.1)\end{array}$ & $\begin{array}{l}1.6 \\
(10.9)\end{array}$ & $\begin{array}{l}16.5 \\
(106.7)\end{array}$ & $\begin{array}{l}25.4 \\
(58.1)\end{array}$ & $\begin{array}{l}1.7 \\
(9.8)\end{array}$ & $\begin{array}{l}7.9 \\
(65.5)\end{array}$ & $\begin{array}{l}23.9 \\
(56.3)\end{array}$ & $\begin{array}{l}1.7 \\
(10.0)\end{array}$ & $\begin{array}{l}9.3 \\
(73.6)\end{array}$ \\
\hline $\begin{array}{l}\text { Web } \\
\text { browsing }\end{array}$ & $\begin{array}{l}2.7 \\
(13.8)\end{array}$ & $\begin{array}{l}0.4 \\
(2.4)\end{array}$ & $\begin{array}{l}0.2 \\
(1.3)\end{array}$ & $\begin{array}{l}4.1 \\
(17.5)\end{array}$ & $\begin{array}{l}0.5 \\
(3.3)\end{array}$ & $\begin{array}{l}0.8 \\
(6.1)\end{array}$ & $\begin{array}{l}3.9 \\
(17.0)\end{array}$ & $\begin{array}{l}0.4 \\
(3.2)\end{array}$ & $\begin{array}{l}0.7 \\
(5.6)\end{array}$ \\
\hline $\begin{array}{l}\text { Games and } \\
\text { comics }\end{array}$ & $\begin{array}{l}89.8 \\
(199.1)\end{array}$ & $\begin{array}{l}4.5 \\
(13.0)\end{array}$ & $\begin{array}{l}7.3 \\
(28.3)\end{array}$ & $\begin{array}{l}24.2 \\
(78.1)\end{array}$ & $\begin{array}{l}1.1 \\
(6.7)\end{array}$ & $\begin{array}{l}2.0 \\
(23.2)\end{array}$ & $\begin{array}{l}34.7 \\
(109.5)\end{array}$ & $\begin{array}{l}1.7 \\
(8.1)\end{array}$ & $\begin{array}{l}2.9 \\
(24.1)\end{array}$ \\
\hline Multimedia & $\begin{array}{l}20.3 \\
(60.7)\end{array}$ & $\begin{array}{l}1.1 \\
(7.0)\end{array}$ & $\begin{array}{l}3.7 \\
(46.3)\end{array}$ & $\begin{array}{l}6.7 \\
(32.0)\end{array}$ & $\begin{array}{l}0.9 \\
(6.5)\end{array}$ & $\begin{array}{l}0.6 \\
(7.6)\end{array}$ & $\begin{array}{l}8.8 \\
(38.3)\end{array}$ & $\begin{array}{l}0.9 \\
(6.6)\end{array}$ & $\begin{array}{l}1.1 \\
(19.7)\end{array}$ \\
\hline Health & $0.0(0.0)$ & $\begin{array}{l}0.0 \\
(0.0)\end{array}$ & $\begin{array}{l}0.0 \\
(0.0)\end{array}$ & $0.4(3.0)$ & $\begin{array}{l}0.0 \\
(0.5)\end{array}$ & $\begin{array}{l}0.0 \\
(0.5)\end{array}$ & $0.3(2.7)$ & $\begin{array}{l}0.0 \\
(0.5)\end{array}$ & $\begin{array}{l}0.0 \\
(0.5)\end{array}$ \\
\hline Camera & $0.6(4.0)$ & $\begin{array}{l}0.0 \\
(0.1)\end{array}$ & $\begin{array}{l}0.0 \\
(0.1)\end{array}$ & $0.2(2.5)$ & $\begin{array}{l}0.0 \\
(0.1)\end{array}$ & $\begin{array}{l}0.0 \\
(0.1)\end{array}$ & $0.3(2.8)$ & $\begin{array}{l}0.0 \\
(0.1)\end{array}$ & $\begin{array}{l}0.0 \\
(0.1)\end{array}$ \\
\hline Total & $\begin{array}{l}207.4 \\
(309.1)\end{array}$ & $\begin{array}{l}12.1 \\
(26.4)\end{array}$ & $\begin{array}{l}39.4 \\
(136.6)\end{array}$ & $\begin{array}{l}158.6 \\
(199.4)\end{array}$ & $\begin{array}{l}10.1 \\
(21.3)\end{array}$ & $\begin{array}{l}27.3 \\
(107.6)\end{array}$ & $\begin{array}{l}166.4 \\
(221.0)\end{array}$ & $\begin{array}{l}10.4 \\
(22.2)\end{array}$ & $\begin{array}{l}29.2 \\
(112.7)\end{array}$ \\
\hline
\end{tabular}

Table 3 shows the multilevel regression results of smartphone usage on sleep quality. Among secondary school students, one minute of daytime smartphone usage was associated with 0.12 minute decrease in total sleeping time that night $(p=0.042,95 \% \mathrm{Cl}:-0.23,-0.007)$. Broken down for different usage purposes, only social network $(\beta=-0.39, p$ $=0.03,95 \% \mathrm{Cl}:-0.73,-0.04)$ was associated with total sleeping time. One minute of bedtime smartphone usage was associated with 0.32 minute increase in wake after sleep onset that night ( $p=0.04,95 \% \mathrm{Cl}: 0.02,0.62)$. One minute of smartphone usage during sleep was associated with sleep efficiency $(\beta=0.013 \%, p=0.01,95 \% \mathrm{Cl}: 0.003 \%, 0.023 \%)$ and WASO ( $\beta=-0.05, p=0.04,95 \% \mathrm{Cl}$ : $-0.10,-0.005)$. Among university students, smartphone usage during daytime, bedtime, and sleep were insignificantly associated with sleep quality. Broken down for different usage purposes, daytime tools usage $(\beta=0.32, p=0.02,95 \% \mathrm{Cl}: 0.04,0.60)$ and bedtime social network usage $(\beta=2.38, p=0.02,95 \%$ $\mathrm{Cl}: 0.37,4.39)$ was associated with total sleeping time, while instant messaging $(\beta=0.04, p=0.048,95 \% \mathrm{Cl}: 0.001$, $0.07)$, web browsing $(\beta=0.47, p=0.02,95 \% \mathrm{Cl}: 0.09,0.84)$, and camera usage $(\beta=25.79, p=0.01,95 \% \mathrm{Cl}: 6.22,45.36)$ during sleep were associated with WASO. 
Table 3

Multilevel regression results of smartphone usage $(\mathrm{min}$ ) on sleep quality (number of participants $=187$, number of days $=814)$

\begin{tabular}{|c|c|c|c|c|c|c|}
\hline & \multicolumn{3}{|c|}{$\begin{array}{l}\text { Secondary school students } \\
\text { (number of participants }=32, \text { number of days }= \\
109 \text { ) }\end{array}$} & \multicolumn{3}{|c|}{$\begin{array}{l}\text { University students } \\
\text { (number of participants }=155 \text {, number of days } \\
=705 \text { ) }\end{array}$} \\
\hline & $\begin{array}{l}\text { Total } \\
\text { sleeping } \\
\text { time (min) }\end{array}$ & $\begin{array}{l}\text { Sleep } \\
\text { efficiency (\%) }\end{array}$ & $\begin{array}{l}\text { Wake after } \\
\text { sleep onset } \\
(\%)\end{array}$ & $\begin{array}{l}\text { Total } \\
\text { sleeping } \\
\text { time (min) }\end{array}$ & $\begin{array}{l}\text { Sleep } \\
\text { efficiency (\%) }\end{array}$ & $\begin{array}{l}\text { Wake after } \\
\text { sleep onset } \\
(\%)\end{array}$ \\
\hline \multicolumn{7}{|l|}{ Daytime } \\
\hline $\begin{array}{l}\text { Social } \\
\text { network }\end{array}$ & $\begin{array}{l}-0.39 *(-0.73 \\
-0.04)\end{array}$ & $\begin{array}{l}-0.0088 \\
(-0.0090 \\
0.0267)\end{array}$ & $\begin{array}{l}-0.04(-0.12 \\
0.04)\end{array}$ & $\begin{array}{l}-0.19(-0.51 \\
0.14)\end{array}$ & $\begin{array}{l}-0.0034 \\
(-0.0166 \\
0.0098)\end{array}$ & $\begin{array}{l}0.004(-0.06 \\
0.05)\end{array}$ \\
\hline $\begin{array}{l}\text { Instant } \\
\text { messaging }\end{array}$ & $\begin{array}{l}0.14(-0.22 \\
0.49)\end{array}$ & $\begin{array}{l}-0.0019 \\
(-0.0175 \\
0.0138)\end{array}$ & $\begin{array}{l}0.04(-0.04 \\
0.11)\end{array}$ & $\begin{array}{l}-0.08(-0.22 \\
0.06)\end{array}$ & $\begin{array}{l}-0.0022 \\
(-0.0080 \\
0.0036)\end{array}$ & $\begin{array}{l}0.01(-0.02 \\
0.03)\end{array}$ \\
\hline Tools & $\begin{array}{l}-0.46(-1.15 \\
0.23\end{array}$ & $\begin{array}{l}-0.0006 \\
(-0.0275 \\
0.0263)\end{array}$ & $\begin{array}{l}-0.04(-0.17 \\
0.09)\end{array}$ & $\begin{array}{l}0.32 * \\
0.60)\end{array}$ & $\begin{array}{l}0.0031 \\
(-0.0083 \\
0.0144)\end{array}$ & $\begin{array}{l}0.01(-0.03 \\
0.06)\end{array}$ \\
\hline $\begin{array}{l}\text { Web } \\
\text { browsing }\end{array}$ & $\begin{array}{l}-1.14(-3.25 \\
0.96)\end{array}$ & $\begin{array}{l}-0.0419 \\
(-0.1238 \\
0.0401)\end{array}$ & $\begin{array}{l}0.17(-0.24 \\
0.57)\end{array}$ & $\begin{array}{l}-0.69(-1.74 \\
0.37)\end{array}$ & $\begin{array}{l}0.0010 \\
(-0.0434 \\
0.0454)\end{array}$ & $\begin{array}{l}-0.05(-0.24 \\
0.13)\end{array}$ \\
\hline $\begin{array}{l}\text { Games } \\
\text { and } \\
\text { comics }\end{array}$ & $\begin{array}{l}-0.17(-0.35 \\
-0.004)\end{array}$ & $\begin{array}{l}-0.0057 \\
(-0.0134 \\
0.0020)\end{array}$ & $\begin{array}{l}0.01(-0.03 \\
0.05)\end{array}$ & $\begin{array}{l}-0.17(-0.37 \\
0.04)\end{array}$ & $\begin{array}{l}-0.0036 \\
(-0.0122 \\
0.0051)\end{array}$ & $\begin{array}{l}0.06(-0.03 \\
0.16)\end{array}$ \\
\hline Multimedia & $\begin{array}{l}-0.44(-0.94 \\
0.06)\end{array}$ & $\begin{array}{l}-0.0090 \\
(-0.0286 \\
0.0105)\end{array}$ & $\begin{array}{l}0.006(-0.09 \\
0.10)\end{array}$ & $\begin{array}{l}0.003(-0.46, \\
0.46)\end{array}$ & $\begin{array}{l}-0.0007 \\
(-0.0192 \\
0.0177)\end{array}$ & $\begin{array}{l}0.01(-0.06 \\
0.09)\end{array}$ \\
\hline Health & $\mathrm{N} / \mathrm{A}$ & N/A & $\mathrm{N} / \mathrm{A}$ & $\begin{array}{l}0.38(-4.38 \\
5.15)\end{array}$ & $\begin{array}{l}0.0568 \\
(-0.1332 \\
0.2469)\end{array}$ & $\begin{array}{l}-0.19(-0.97 \\
0.59)\end{array}$ \\
\hline Camera & $\begin{array}{l}3.20(-4.96 \\
11.37)\end{array}$ & $\begin{array}{l}-0.0129 \\
(-0.3191 \\
0.2932)\end{array}$ & $\begin{array}{l}0.02(-1.43 \\
1.48)\end{array}$ & $\begin{array}{l}0.02(-5.54 \\
5.58)\end{array}$ & $\begin{array}{l}0.0568 \\
(-0.1592 \\
0.2727)\end{array}$ & $\begin{array}{l}-0.22(-1.10 \\
0.66)\end{array}$ \\
\hline Total & $\begin{array}{l}-0.12 *(-0.23 \\
-0.007)\end{array}$ & $\begin{array}{l}-0.0029 \\
(-0.0077 \\
0.0019)\end{array}$ & $\begin{array}{l}0.01(-0.02 \\
0.03)\end{array}$ & $\begin{array}{l}-0.04(-0.13 \\
0.04)\end{array}$ & $\begin{array}{l}-0.0012 \\
(-0.0046 \\
0.0022)\end{array}$ & $\begin{array}{l}0.01(-0.01 \\
0.02)\end{array}$ \\
\hline \multicolumn{7}{|l|}{ Bedtime } \\
\hline $\begin{array}{l}\text { Social } \\
\text { network }\end{array}$ & $\begin{array}{l}1.10(-5.35 \\
7.55)\end{array}$ & $\begin{array}{l}-0.1152 \\
(-0.3542 \\
0.1239)\end{array}$ & $\begin{array}{l}0.45(-0.73 \\
1.64)\end{array}$ & $\begin{array}{l}2.38 *(0.37 \\
4.39)\end{array}$ & $\begin{array}{l}-0.0022 \\
(-0.0831 \\
0.0787)\end{array}$ & $\begin{array}{l}0.15(-0.18 \\
0.48)\end{array}$ \\
\hline $\begin{array}{l}\text { Instant } \\
\text { messaging }\end{array}$ & $\begin{array}{l}1.77(-1.38 \\
4.92)\end{array}$ & $\begin{array}{l}-0.1231 \\
(-0.2512 \\
0.0050)\end{array}$ & $\begin{array}{l}0.64 * \\
1.25)\end{array}$ & $\begin{array}{l}0.24(-1.23 \\
1.70)\end{array}$ & $\begin{array}{l}0.0456 \\
(-0.0122, \\
0.1033)\end{array}$ & $\begin{array}{l}-0.18(-0.42 \\
0.06)\end{array}$ \\
\hline Tools & $\begin{array}{l}-4.34(-10.25 \\
1.56)\end{array}$ & $\begin{array}{l}0.1062 \\
(-0.1155 \\
0.3280)\end{array}$ & $\begin{array}{l}-0.95(-2.04 \\
0.14)\end{array}$ & $\begin{array}{l}-0.86(-3.25 \\
1.54)\end{array}$ & $\begin{array}{l}-0.0460 \\
(-0.1393 \\
0.0473)\end{array}$ & $\begin{array}{l}0.13(-0.25 \\
0.51)\end{array}$ \\
\hline
\end{tabular}




\begin{tabular}{|c|c|c|c|c|c|c|}
\hline \multirow[b]{2}{*}{$\begin{array}{l}\text { Web } \\
\text { browsing }\end{array}$} & \multicolumn{3}{|c|}{$\begin{array}{l}\text { Secondary school students } \\
\text { (number of participants }=32 \text {, number of days }= \\
109 \text { ) }\end{array}$} & \multicolumn{3}{|c|}{$\begin{array}{l}\text { University students } \\
\text { (number of participants }=155, \text { number of days } \\
=705 \text { ) }\end{array}$} \\
\hline & $\begin{array}{l}-3.06(-9.35 \\
15.46)\end{array}$ & $\begin{array}{l}0.2611 \\
(-0.1877 \\
0.7080)\end{array}$ & $\begin{array}{l}-0.94(-3.18 \\
1.30)\end{array}$ & $\begin{array}{l}-1.44(-6.24 \\
3.36)\end{array}$ & $\begin{array}{l}0.1174 \\
(-0.0709 \\
0.3058)\end{array}$ & $\begin{array}{l}-0.32(-1.09 \\
0.45)\end{array}$ \\
\hline $\begin{array}{l}\text { Games } \\
\text { and } \\
\text { comics }\end{array}$ & $\begin{array}{l}0.42(-2.17 \\
3.01)\end{array}$ & $\begin{array}{l}-0.0802 \\
(-0.1835 \\
0.0232)\end{array}$ & $\begin{array}{l}0.45(-0.06 \\
0.96)\end{array}$ & $\begin{array}{l}1.75(-0.44 \\
3.94)\end{array}$ & $\begin{array}{l}0.0128 \\
(-0.0735 \\
0.0991)\end{array}$ & $\begin{array}{l}0.09(-0.27 \\
0.44)\end{array}$ \\
\hline Multimedia & $\begin{array}{l}-4.00(-10.78 \\
2.78)\end{array}$ & $\begin{array}{l}-0.0100 \\
(-0.2614 \\
0.2414)\end{array}$ & $\begin{array}{l}-0.10(-1.35 \\
1.16)\end{array}$ & $\begin{array}{l}0.89(-1.34 \\
3.11)\end{array}$ & $\begin{array}{l}-0.0233 \\
(-0.1099 \\
0.0633)\end{array}$ & $\begin{array}{l}0.19(-0.16 \\
0.54)\end{array}$ \\
\hline Health & N/A & N/A & $\mathrm{N} / \mathrm{A}$ & $\begin{array}{l}17.22(-8.78 \\
43.22)\end{array}$ & $\begin{array}{l}0.5519 \\
(-0.4686 \\
1.5720)\end{array}$ & $\begin{array}{l}-1.98(-6.17 \\
2.21)\end{array}$ \\
\hline Camera & $\begin{array}{l}-135.36 \\
(-601.04 \\
330.32)\end{array}$ & $\begin{array}{l}6.1200 \\
(-11.1420 \\
23.3880)\end{array}$ & $\begin{array}{l}-32.09 \\
(-114.94 \\
50.76)\end{array}$ & $\begin{array}{l}-116.63 \\
(-251.81 \\
18.54)\end{array}$ & $\begin{array}{l}3.6540 \\
(-1.6020 \\
8.910)\end{array}$ & $\begin{array}{l}-12.45(-33.91, \\
9.01)\end{array}$ \\
\hline Total & $\begin{array}{l}0.59 \text { (-0.96, } \\
2.13)\end{array}$ & $\begin{array}{l}-0.0604 \\
(-0.1219 \\
0.0010)\end{array}$ & $\begin{array}{l}0.32 * \\
0.62)\end{array}$ & $\begin{array}{l}0.68(-0.18 \\
1.54)\end{array}$ & $\begin{array}{l}0.0153 \\
(-0.0188 \\
0.0494)\end{array}$ & $\begin{array}{l}-0.01 \\
0.13)\end{array}(-0.15$ \\
\hline \multicolumn{7}{|l|}{$\begin{array}{l}\text { During } \\
\text { sleep }\end{array}$} \\
\hline $\begin{array}{l}\text { Social } \\
\text { network }\end{array}$ & $\begin{array}{l}1.82(-0.91 \\
4.55)\end{array}$ & $\begin{array}{l}0.0433 \\
(-0.0634 \\
0.1499)\end{array}$ & $\begin{array}{l}-0.08(-0.60 \\
0.45)\end{array}$ & $\begin{array}{l}-0.02(-0.63 \\
0.59)\end{array}$ & $\begin{array}{l}-0.0059 \\
(-0.0297 \\
0.0179)\end{array}$ & $\begin{array}{l}0.02(-0.07 \\
0.12)\end{array}$ \\
\hline $\begin{array}{l}\text { Instant } \\
\text { messaging }\end{array}$ & $\begin{array}{l}0.26(-0.54 \\
1.07)\end{array}$ & $\begin{array}{l}0.0119 \\
(-0.0290 \\
0.0528)\end{array}$ & $\begin{array}{l}-0.06(-0.24 \\
0.13)\end{array}$ & $\begin{array}{l}0.07(-0.16, \\
0.29)\end{array}$ & $\begin{array}{l}-0.0069 \\
(-0.0157 \\
0.0020)\end{array}$ & $\begin{array}{l}0.04 *(0.001 \\
0.07)\end{array}$ \\
\hline Tools & $\begin{array}{l}0.60(-0.01 \\
1.22)\end{array}$ & $\begin{array}{l}-0.0018 \\
(-0.0249 \\
0.0213)\end{array}$ & $\begin{array}{l}0.06(-0.05 \\
0.18)\end{array}$ & $\begin{array}{l}0.04(-0.30 \\
0.39)\end{array}$ & $\begin{array}{l}0.0062 \\
(-0.0074 \\
0.0197)\end{array}$ & $\begin{array}{l}-0.02(-0.08 \\
0.03)\end{array}$ \\
\hline $\begin{array}{l}\text { Web } \\
\text { browsing }\end{array}$ & $\begin{array}{l}12.67 \\
(-10.03 \\
35.37)\end{array}$ & $\begin{array}{l}-0.0062 \\
(-1.2060 \\
1.1940)\end{array}$ & $\begin{array}{l}0.53(-4.47 \\
5.86)\end{array}$ & $\begin{array}{l}2.13(-0.19 \\
4.46)\end{array}$ & $\begin{array}{l}-0.0740 \\
(-0.1655 \\
0.0175)\end{array}$ & $\begin{array}{l}0.47 *(0.09 \\
0.84)\end{array}$ \\
\hline $\begin{array}{l}\text { Games } \\
\text { and } \\
\text { comics }\end{array}$ & $\begin{array}{l}0.84(-0.18 \\
1.86)\end{array}$ & $\begin{array}{l}0.0095 \\
(-0.0298 \\
0.0489)\end{array}$ & $\begin{array}{l}0.02(-0.17 \\
0.21)\end{array}$ & $\begin{array}{l}0.25(-0.34 \\
0.83)\end{array}$ & $\begin{array}{l}-0.0125 \\
(-0.0352 \\
0.0102)\end{array}$ & $\begin{array}{l}0.06(-0.03 \\
0.16)\end{array}$ \\
\hline Multimedia & $\begin{array}{l}0.13(-0.89 \\
1.16)\end{array}$ & $\begin{array}{l}0.0076 \\
(-0.0350 \\
0.0501)\end{array}$ & $\begin{array}{l}-0.03(-0.23 \\
0.17)\end{array}$ & $\begin{array}{l}1.74(-0.06 \\
3.54)\end{array}$ & $\begin{array}{l}-0.0291 \\
(-0.0992 \\
0.0409)\end{array}$ & $\begin{array}{l}0.28(-0.01 \\
0.57)\end{array}$ \\
\hline Health & $\mathrm{N} / \mathrm{A}$ & $\mathrm{N} / \mathrm{A}$ & N/A & $\begin{array}{l}6.39(-20.66, \\
33.45)\end{array}$ & $\begin{array}{l}0.9240 \\
(-0.1309 \\
1.9740)\end{array}$ & $\begin{array}{l}-3.64(-7.95 \\
0.68)\end{array}$ \\
\hline Camera & $\begin{array}{l}286.27 \\
(-136.42 \\
708.96)\end{array}$ & $\begin{array}{l}-13.5600 \\
(-29.1660 \\
2.0460)\end{array}$ & $\begin{array}{l}97.43^{\star}(23.21 \\
171.65)\end{array}$ & $\begin{array}{l}\text { 157.39* } \\
(37.41 \\
277.37)\end{array}$ & $\begin{array}{l}-3.4020 \\
(-8.1900 \\
1.3920)\end{array}$ & $\begin{array}{l}25.79 *(6.22 \\
45.36)\end{array}$ \\
\hline
\end{tabular}




\begin{tabular}{|c|c|c|c|c|c|c|}
\hline \multirow[b]{2}{*}{ Total } & \multicolumn{3}{|c|}{$\begin{array}{l}\text { Secondary school students } \\
\text { (number of participants }=32 \text {, number of days = } \\
109 \text { ) }\end{array}$} & \multicolumn{3}{|c|}{$\begin{array}{l}\text { University students } \\
\text { (number of participants }=155 \text {, number of days } \\
=705 \text { ) }\end{array}$} \\
\hline & $\begin{array}{l}0.11(-0.16 \\
0.37)\end{array}$ & $\begin{array}{l}0.0130 \star \\
(0.0028 \\
0.0232)\end{array}$ & $\begin{array}{l}-0.05^{\star}(-0.10 \\
-0.005)\end{array}$ & $\begin{array}{l}0.02(-0.13 \\
0.18)\end{array}$ & $\begin{array}{l}-0.0038 \\
(-0.0098 \\
0.0023)\end{array}$ & $\begin{array}{l}0.02(-0.01 \\
0.04)\end{array}$ \\
\hline
\end{tabular}

Table 4 shows the multilevel regression results of smartphone usage on physical activity level. One minute of daytime smartphone usage was associated with 7.15 steps increase in the number of steps $(p=0.02,95 \% \mathrm{Cl}: 1.02,13.28)$ among secondary school students and 3.52 steps increase in the number of steps $(p=0.03,95 \% \mathrm{Cl}: 0.37,6.66)$ among university students on the next day. Social network, instant messaging, tools, and multimedia usage were positively associated with the time spent on MVPA. Smartphone usage during bedtime and sleep were insignificantly associated with physical activity level, except web browsing during sleep was associated with number of steps $(\beta=1153.07, p=$ $0.04,95 \%$ Cl: $57.92,2248.22)$ among secondary school students. Table 5 shows that, for both secondary school students and university students, better sleep quality was associated with higher volume of physical activity level on the next day, except that one percent increase in sleep efficiency was associated with 2.29 minutes decrease in time spent on MVPA ( $p=0.02,95 \% \mathrm{Cl}: 0.34,4.25)$ among secondary school students. 
Table 4

Multilevel regression results of smartphone usage ( $\mathrm{min}$ ) on physical activity level (number of participants $=187$, number of days $=1,106$ )

\begin{tabular}{|c|c|c|c|c|}
\hline & \multicolumn{2}{|c|}{$\begin{array}{l}\text { Secondary school students } \\
\text { (number of participants }=32, \text { number of days }= \\
176 \text { ) }\end{array}$} & \multicolumn{2}{|c|}{$\begin{array}{l}\text { University students } \\
\text { (number of participants = 155, number of days } \\
=930 \text { ) }\end{array}$} \\
\hline & Number of steps & $\begin{array}{l}\text { Moderate-to-vigorous } \\
\text { physical activity (min) }\end{array}$ & Number of steps & $\begin{array}{l}\text { Moderate-to-vigorous } \\
\text { physical activity (min) }\end{array}$ \\
\hline \multicolumn{5}{|l|}{ Daytime } \\
\hline $\begin{array}{l}\text { Social } \\
\text { network }\end{array}$ & $\begin{array}{l}10.49(-11.04 \\
32.02)\end{array}$ & $0.12(-0.27,0.51)$ & $\begin{array}{l}13.52 *(0.94 \\
26.11)\end{array}$ & $0.27 *(0.06,0.48)$ \\
\hline $\begin{array}{l}\text { Instant } \\
\text { messaging }\end{array}$ & $\begin{array}{l}25.89 *(5.96 \\
45.82)\end{array}$ & $0.37 *(0.01,0.72)$ & $\begin{array}{l}6.46 *(1.03 \\
11.90)\end{array}$ & $0.09(-0.01,0.18)$ \\
\hline Tools & $\begin{array}{l}32.50(-4.02 \\
69.01)\end{array}$ & $0.72 *(0.08,1.36)$ & $\begin{array}{l}1.27(-6.94 \\
14.16)\end{array}$ & $-0.02(-0.21,0.16)$ \\
\hline $\begin{array}{l}\text { Web } \\
\text { browsing }\end{array}$ & $\begin{array}{l}77.94(-32.26 \\
188.13)\end{array}$ & $0.94(-0.98,2.88)$ & $\begin{array}{l}22.77(-19.82 \\
65.37)\end{array}$ & $0.29(-0.46,1.03)$ \\
\hline $\begin{array}{l}\text { Games } \\
\text { and } \\
\text { comics }\end{array}$ & $5.35(-4.76,15.45)$ & $-0.001(-0.18,0.18)$ & $0.77(-7.55,9.08)$ & $0.02(-0.12,0.16)$ \\
\hline Multimedia & $\begin{array}{l}25.69 *(0.11 \\
51.26)\end{array}$ & $0.50 *(0.05,0.94)$ & $\begin{array}{l}7.27(-10.87 \\
25.41)\end{array}$ & $0.14(-0.16,0.43)$ \\
\hline Health & $\mathrm{N} / \mathrm{A}$ & $\mathrm{N} / \mathrm{A}$ & $\begin{array}{l}-9.45(-198.77 \\
179.87)\end{array}$ & $-0.04(-3.15,3.06)$ \\
\hline Camera & $\begin{array}{l}-100.56(-500.55 \\
299.42)\end{array}$ & $-1.96(-8.93,5.01)$ & $\begin{array}{l}17.52(-200.06 \\
235.10)\end{array}$ & $0.17(-3.34,3.68)$ \\
\hline Total & $7.15^{\star}(1.02,13.28)$ & $0.09(-0.02,0.20)$ & $\begin{array}{l}3.52 *(0.37 \\
6.66)\end{array}$ & $0.05(-0.001,0.11)$ \\
\hline \multicolumn{5}{|l|}{ Bedtime } \\
\hline $\begin{array}{l}\text { Social } \\
\text { network }\end{array}$ & $\begin{array}{l}-88.62(-400.75 \\
223.50)\end{array}$ & $-1.28(-6.74,4.17)$ & $\begin{array}{l}-52.87(-130.75 \\
25.01)\end{array}$ & $-0.48(-1.75,0.80)$ \\
\hline $\begin{array}{l}\text { Instant } \\
\text { messaging }\end{array}$ & $\begin{array}{l}-66.99(-233.06 \\
99.08)\end{array}$ & $-1.24(-4.17,1.68)$ & $\begin{array}{l}-25.11(-82.23 \\
32.01)\end{array}$ & $0.34(-0.59,1.27)$ \\
\hline Tools & $\begin{array}{l}190.96(-108.27 \\
490.19)\end{array}$ & $4.12(-1.06,9.31)$ & $\begin{array}{l}-57.54(-152.49 \\
37.40)\end{array}$ & $-0.93(-2.46,0.60)$ \\
\hline $\begin{array}{l}\text { Web } \\
\text { browsing }\end{array}$ & $\begin{array}{l}148.88(-469.21 \\
766.97)\end{array}$ & $4.40(-6.36,15.15)$ & $\begin{array}{l}-29.05(-217.86 \\
159.76)\end{array}$ & $0.07(-3.01,3.15)$ \\
\hline $\begin{array}{l}\text { Games } \\
\text { and } \\
\text { comics }\end{array}$ & $\begin{array}{l}-58.44(-193.35 \\
76.46)\end{array}$ & $-0.26(-2.60,2.15)$ & $\begin{array}{l}-48.06(-134.14 \\
38.01)\end{array}$ & $-1.11(-2.52,0.31)$ \\
\hline Multimedia & $\begin{array}{l}-3.92(-336.54 \\
328.71)\end{array}$ & $2.03(-3.74,7.81)$ & $\begin{array}{l}10.16(-76.11 \\
96.43)\end{array}$ & $0.17(-1.22,1.56)$ \\
\hline Health & $\mathrm{N} / \mathrm{A}$ & $\mathrm{N} / \mathrm{A}$ & $\begin{array}{l}-665.89 \\
(-1683.57 \\
351.78)\end{array}$ & $-11.01(-27.43,5.40)$ \\
\hline
\end{tabular}




\begin{tabular}{|c|c|c|c|c|}
\hline \multirow[b]{2}{*}{ Camera } & \multicolumn{2}{|c|}{$\begin{array}{l}\text { Secondary school students } \\
\text { (number of participants }=32 \text {, number of days }= \\
176 \text { ) }\end{array}$} & \multicolumn{2}{|c|}{$\begin{array}{l}\text { University students } \\
\text { (number of participants = } 155 \text {, number of days } \\
=930 \text { ) }\end{array}$} \\
\hline & $\begin{array}{l}5238.15 \\
(-19576.76 \\
30053.05)\end{array}$ & $65.51(-368.41,499.43)$ & $\begin{array}{l}-3562.48 \\
(-8812.56 \\
1687.60)\end{array}$ & $-44.60(-129.43,40.23)$ \\
\hline Total & $\begin{array}{l}-13.78(-94.63 \\
67.07)\end{array}$ & $0.10(-1.32,1.52)$ & $\begin{array}{l}-26.24(-59.56 \\
7.08)\end{array}$ & $-0.13(-0.68,0.41)$ \\
\hline \multicolumn{5}{|l|}{$\begin{array}{l}\text { During } \\
\text { sleep }\end{array}$} \\
\hline $\begin{array}{l}\text { Social } \\
\text { network }\end{array}$ & $\begin{array}{l}39.49(-111.49 \\
190.47)\end{array}$ & $-0.01(-2.66,2.65)$ & $\begin{array}{l}2.97(-20.79 \\
26.72)\end{array}$ & $0.01(-0.38,0.39)$ \\
\hline $\begin{array}{l}\text { Instant } \\
\text { messaging }\end{array}$ & $\begin{array}{l}29.84(-12.52 \\
72.21)\end{array}$ & $0.65(-0.10,1.40)$ & $\begin{array}{l}6.27(-2.45 \\
14.99)\end{array}$ & $0.02(-0.13,0.16)$ \\
\hline Tools & $\begin{array}{l}-20.04(-50.82 \\
10.75)\end{array}$ & $-0.38(-0.92,0.15)$ & $\begin{array}{l}7.16(-6.54 \\
20.86)\end{array}$ & $0.06(-0.16,0.28)$ \\
\hline $\begin{array}{l}\text { Web } \\
\text { browsing }\end{array}$ & $\begin{array}{l}1153.07 * \\
2248.22)\end{array}$ & $1.04(-1.94,4.03)$ & $\begin{array}{l}-82.60(-173.48 \\
8.29)\end{array}$ & $-1.24(-2.72,0.24)$ \\
\hline $\begin{array}{l}\text { Games } \\
\text { and } \\
\text { comics }\end{array}$ & $\begin{array}{l}31.90(-19.90 \\
83.70)\end{array}$ & $0.65(-0.25,1.55)$ & $\begin{array}{l}-12.29(-35.11 \\
10.53)\end{array}$ & $-0.15(-0.51,0.22)$ \\
\hline Multimedia & $\begin{array}{l}21.64(-28.92 \\
72.20)\end{array}$ & $-0.12(-1.00,0.76)$ & $\begin{array}{l}-41.90(-112.46 \\
28.67)\end{array}$ & $-0.64(-1.78,0.50)$ \\
\hline Health & $\mathrm{N} / \mathrm{A}$ & $\mathrm{N} / \mathrm{A}$ & $\begin{array}{l}9.40(-1048.04 \\
1066.83)\end{array}$ & $-1.95(-19.01,15.12)$ \\
\hline Camera & $\begin{array}{l}-3407.19 \\
(-23839.59 \\
17025.20)\end{array}$ & $-83.94(-439.96,272.08)$ & $\begin{array}{l}-1432.84 \\
(-6071.19 \\
3205.02)\end{array}$ & $-18.54(-94.34,57.27)$ \\
\hline Total & $6.87(-6.90,20.63)$ & $0.09(-0.15,0.33)$ & $3.42(-2.58,9.42)$ & $-0.003(-0.10,0.09)$ \\
\hline
\end{tabular}

Table 5

Multilevel regression results of sleep quality on physical activity level (number of participants $=352$, number of days $=$ $1,517)$

\begin{tabular}{|c|c|c|c|c|}
\hline & \multicolumn{2}{|c|}{$\begin{array}{l}\text { Secondary school students } \\
\text { (number of participants }=123 \text {, number of } \\
\text { days }=459 \text { ) }\end{array}$} & \multicolumn{2}{|c|}{$\begin{array}{l}\text { University students } \\
\text { (number of participants }=229, \text { number of } \\
\text { days }=1,058 \text { ) }\end{array}$} \\
\hline & $\begin{array}{l}\text { Number of } \\
\text { steps }\end{array}$ & $\begin{array}{l}\text { Moderate-to-vigorous } \\
\text { physical activity (min) }\end{array}$ & $\begin{array}{l}\text { Number of } \\
\text { steps }\end{array}$ & $\begin{array}{l}\text { Moderate-to-vigorous } \\
\text { physical activity (min) }\end{array}$ \\
\hline $\begin{array}{l}\text { Total sleeping } \\
\text { time (hr) }\end{array}$ & $\begin{array}{l}-706.06 * \star \star \\
(-932.24 \\
-479.89)\end{array}$ & $-10.83^{\star \star \star}(-15.12,-6.54)$ & $\begin{array}{l}-569.76 \star \star \star \\
(-700.71 \\
-438.80)\end{array}$ & $-7.95^{\star \star \star}(-10.16,-5.74)$ \\
\hline $\begin{array}{l}\text { Sleep efficiency } \\
(\%)\end{array}$ & $\begin{array}{l}-112.44 *(12.55 \\
212.33)\end{array}$ & $2.29 *(0.34,4.25)$ & $\begin{array}{l}-1.82(-62.24 \\
58.61)\end{array}$ & $-0.40(-1.41,0.61)$ \\
\hline $\begin{array}{l}\text { Wake after } \\
\text { sleep onset } \\
\text { (min) }\end{array}$ & $\begin{array}{l}-42.74 * \star \star \\
(-65.12,-20.37)\end{array}$ & $-0.76 *(-1.19,-0.33)$ & $\begin{array}{l}-15.28 *(-29.15 \\
-1.42)\end{array}$ & $-0.13(-0.36,0.10)$ \\
\hline
\end{tabular}




\section{Discussion}

We conducted a study to examine the association between objectively-measured smartphone usage, sleep quality, and physical activity level among Hong Kong Chinese adolescents and young adults. There were several advantages to using the objective measurement of smartphone usage. First, we could identify different purposes for which smartphone was being used, and showed the effect of smartphone use on different aspects of life. For instance, we found that only the use of social network apps and games and comics apps was associated with total sleeping time. Second, together with sleeping parameters objectively measured with accelerometer, we could define sleeping time and bedtime smartphone usage objectively, and exclude the possibility of reverse causation, whereby people with sleep problems would use their smartphone during bedtime as a way of spending their energy [24]. Third, while the validity of self-reported smartphone usage was questionable, the source of measurement error could be eliminated through objective measurement of smartphone usage.

In studies on the association between smartphone usage and sleep quality, most used a self-reported questionnaire to measure sleep quality and only three studies measured sleep quality with a wrist-worn accelerometer [25-27]. Surprisingly, all three studies found no association. On the one hand, the finding of a null association might be a true finding; on the other hand, it is possible that these studies committed a type II error because the sample sizes ranged from 55 to 110 only. With a repeated measure of 814 nights in a study involving 187 subjects, ours has the largest in sample size and showed that both daytime and bedtime smartphone usage were associated with total sleeping time but not with sleep efficiency and WASO.

Several researchers attempted to differentiate between the physiological effects of daytime and bedtime smartphone usage. Compared to other devices with electronic screens such as televisions and video game consoles, the smartphone is more commonly used in bed as they are portable and are usually placed very close to the user $[28,29]$. To the best of our knowledge, all existing studies have relied on self-reported data to determine bedtime smartphone usage. As the validity of self-reports of bedtime smartphone usage requires the accurate recalling of both bedtime and smartphone usage, we questioned the validity of self-report and used objective measure of sleep onset and smartphone usage to determine bedtime smartphone usage. The invalidity of self-reported bedtime smartphone usage is shown by the disagreement of our results with other existing self-reported results; for example a Taiwanese study using self-reported data showed that bedtime smartphone usage was very common (95\%) among junior college students [30], but the current study recorded such usage in less than half of the nights (42.4\%). Our results also highlighted the importance of distinguishing between daytime and bedtime smartphone usage, as total sleeping time was negatively related to daytime usage but not related to bedtime usage. We found no association between smartphone usage, both daytime and bedtime, and sleeping efficiency, and these results did not agree with some previous findings [31-34]. We believe that the effects of smartphone usage and sleep outcomes are still controversial (some other studies found a null association [35]) and warrant further investigations, especially through employing well-designed controlled trials. We suspect that, in previous studies, participants might mixed up bedtime smartphone usage and usage during sleep, as we found that smartphone usage during sleep did improve sleep efficiency. To the best of our knowledge, very few randomized controlled trials have been conducted in this direction, and some found a null association between smartphone usage and sleep outcomes due to small sample size [36].

Besides the time of use, the purpose for which a smartphone is used may also have an impact on sleep outcomes. This study found that only using a smartphone for social network apps and games and comics apps (with marginallysignificant $p$-value of 0.053 among secondary school students), but not for other types of apps, was associated with total sleeping time. It was hypothesized that interactive smartphone use should have stronger effects on sleep than the passive viewing of a smartphone screen [37]. Our results only partially agreed with this hypothesis. Social network 
app usage can be regarded as passive viewing as its use does not involve frequent touching of the phone. However, the contents of social network are updated extremely quickly and new updates can be expected to occur every minute. Therefore, users may be anxious about missing out on new content, and such anxiety may cause users to stay awake during the night [38]. Yet, some studies found that social network use was, in fact, positively related to sleep quality [39], partially because social network sites can help users to connect, make new friends, and share their stress [39]. In addition to social network apps, instant messaging apps were also commonly used among our subjects, and we found no association between its use and all sleep outcomes. It was hypothesized that it is difficult to top oneself from messaging, which thereby delays sleep onset, and this was supported by studies showed that texting was negatively associated with sleep quality [40] and its effect was the strongest on sleep duration among other electronic screen usages [41]. However, we questioned the validity of these results as they relied on self-reported time spent on instant messaging and such data, being recalled data, is inherently unreliable. Our data showed that instant messaging apps were used frequently, at an average of 363.7 times per day and that each session only lasted for an average of 1.7 minutes. We believe that the frequent and short usage nature of instant messaging apps made the recalling of time spent on them challenging.

It was generally expected that the use of a smartphone would lead to a sedentary and inactive lifestyle due to its sedentary nature $[7,42]$. Surprisingly, our data showed that social network, instant messaging, tools, and multimedia app usage at daytime were all positively associated with physical activity level. We believe that there was a bidirectional causation. Those who were more physically active might have spent more time on social network and instant messaging apps to share their photos and information. Similarly, some of the multimedia app usage might have been occurred concurrently with physical activity (e.g., playing a video during jogging on a treadmill). On the other hand, our data found no association between games and health app usage and physical activity level. Recently, some augmented reality games developed for smartphone have encouraged walking in the real world (e.g., Pokémon GO), and there is evidence to show that their use is promoting engagement in physical activity $[43,44]$. In the current study, too few subjects played Pokémon GO (11 out of 187, 5.9\%); therefore, the health-promoting effect of this kind of augmented reality game might have been diluted by the sedentary effect of other kinds of games. Similarly, users of health-related apps, for instance pedometer apps, were found to be physically more active than non-users $[45,46]$. In the current study, too few subjects used health-related apps (31 out of 187, 16.6\%); less than the previously reported percentage (34.1\%) in a US sample [46]. Most importantly, no secondary school students in our sample used any kind of health-related apps during the measurement period, therefore, we were unable to detect the associations between the usage of health-related apps, sleep quality, and physical activity level.

The major limitation of this study lied in the area of sampling and data collection. Only Android users were recruited in this study and cautions should be taken when our results are generalized to users of other smartphones. This also explains the small number of adolescent participants in our sample; most of the students in our recruited secondary schools were using an iPhone that is not supported by our tracking app. In our sample, about 10\% of the participants owned more than one smartphone. Since the smartphone usage tracking app was only installed in one smartphone of the subjects, we might have underestimated the total smartphone usage. Another limitation was that our smartphone tracking app could not record the usage of some Android default system apps, for example Google Chrome, the default web browser of Android system, in some versions of Android operating system. A minor limitation was that different recruitment approach was used in secondary school and universities that might affect the generalizability of our results.

\section{Conclusions}


Time spent on social network apps and games and comics apps was associated with total sleeping time, and time spent on social network apps, instant messaging apps, and multimedia apps were associated with the number of steps and MVPA among Hong Kong Chinese adolescents and young adults.

\section{Declarations}

\section{Availability of data and materials}

The dataset(s) supporting the conclusions of this article is(are) available upon request.

\section{Acknowledgments}

The authors would like to thank Mr Ming Wong (Hong Kong Polytechnic University) for data collection and data management of the current study.

\section{Ethics approval and consent to participate}

Written consent was obtained from all of the participants. For participants under the age of 18, parental written consent was also obtained. This study was approved by the Human Subjects Ethics Sub-Committee of the Hong Kong Polytechnic University.

\section{Authors' contribution}

Dr Paul $\mathrm{H}$. Lee designed the study, obtained the funding, supervised the data collection, conducted the data analysis, and drafted the manuscript. Dr Andy C. Y. Tse, Dr Cynthia S. T. Wu, and Dr Yim Wah Mak supervised the data collection, managed the research assistants, and critically reviewed the manuscript. Dr Uichin Lee provided critical feedback on data analysis and reviewed the manuscript. All author approved the final version of the manuscript.

\section{Consent for publication}

Not applicable.

\section{Competing interests}

The authors declare that there are no known conflicts of interest associated with this publication.

\section{Funding}

This study was funded by the General Research Fund Early Career Scheme (Ref: PolyU 251056/16M) from the University Grants Committee of the Hong Kong Special Administrative Region, China.

\section{References}


1. Number of smartphone users worldwide from 2014 to 2020 (in billions)

[http://www.statista.com/statistics/330695/number-of-smartphone-users-worldwide/]

2. Kwon M, Kim DJ, Cho H, Yang S: The Smartphone Addiction Scale: Development and validation of a short version for adolescents. PLoS One 2013, 8(12):e83558.

3. Mobile technology fact sheet [http://www.pewinternet.org/fact-sheets/mobile-technology-fact-sheet/]

4. The 15 Countries With the Highest Smartphone Penetration [https://mashable.com/2013/08/27/globalsmartphone-penetration/]

5. Health Effects on Use of Internet and Electronic Screen Products

[http://www.studenthealth.gov.hk/english/internet/health_effects.html]

6. Hong Kong Polytechnic University Department of Physiotherapy: A survey on health effects of the use of smart phones and related electronic products in Hong Kong. In.; 2013.

7. Lepp A, Barkley JE, Sanders GJ, Rebold M, Gates P: The relationship between cell phone use, physical and sedentary activity, and cardiorespiratory fitness in a sample of U.S. college students. Int J Behav Nutr Phys Act $2013,10: 79$.

8. Punamaki R-L, Wallenius M, Nygard C-H, Saarni L, Rimpela A: Use of information and communication technology (ICT) and perceived health in adolescence: The role of sleeping habits and waking-time tiredness. $J$ Adolescence 2007, 30(4):569-585.

9. Hysing M, Pallesen S, Stormark KM, Jakobsen R, Lundervold AJ, Sivertsen B: Sleep and use of electronic devices in adolescence: results from a large population-based study. BMJ Open 2015, 5:e006748.

10. Benson VS, Pirie K, Schuz J, Reeves GK, Beral V, Green J, for the Million Women Study Collaborators: Mobile phone use and risk of brain neoplasms and other cancers: prospective study. Int J Epidemio/ 2013, 42(3):792-802.

11. Bohmer M, Hecht B, Schoning J, Kruger A, Bauer G: Falling asleep with Angry Birds, Facebook and Kindle - A large scale study on mobile application usage. In: MobileHCl 2011: 2011; Stockholm, Sweden; 2011.

12. Do TMT, Blom J, Gatica-Perez D: Smartphone usage in the wild: a large-scale analysis of applications and context. In: ICMI'11: 2011; Alicante, Spain; 2011.

13. Falaki H, Mahajan R, Estrin D: SystemSens: A tool for monitoring usage in smartphone research deployments. In: MobiArch'11: 2011; Bethesda, Maryland, USA; 2011: 25-30.

14. Falaki H, Mahajan R, Kandula S, Lymberopoulos D, Govindan R, Estrin D: Diversity in smartphone usage. In: MobiSys'10: 2010; San Francisco, California, USA; 2010: 179-194.

15. Shye A, Scholbrock B, Memik G, Dinda PA: Characterizing and modeling user activity on smartphones: Summary. In: SIGMETRICS'10: 2010; New York, USA; 2010: 375-376.

16. Soikkeli T, Karikoski J, Hammainen H: Diversity and end user context in smartphone usage sessions. In: Fifth International Conference on Next Generation Mobile Applications and Services: 2011; 2011: 7-12.

17. Chittaranjan G, Blom J, Gatica-Perez D: Mining large-scale smartphone data for personality studies. Pers Ubiquit Comput 2013, 17(3):433-450.

18. Ryde GC, Gilson ND, Suppini A, Brown WJ: Validation of a novel, objective measure of occupational sitting. J Occup Health 2012, 54:383-386.

19. Pfitzner R, Gorzelniak L, Heinrich J, Von Berg A, Klumper C, Bauer CP, Koletzko S, Berdel D, Horsch A, Schulz H et al: Physical activity in German adolescents measured by accelerometry and activity diary: Introducing a comprehensive approach for data management and preliminary results. PLoS One 2013, 8(6):e65192.

20. Lee PH, Suen LKP: The convergent validity of Actiwatch 2 and ActiGraph Link accelerometers in measuring total sleeping period, wake after sleep onset, and sleep efficiency in free-living condition. Sleep Breath 2017, 21(1):209- 
215.

21. Toon E, Davey MJ, Hollis SL, Nixon GM, Horne RSC, Biggs SN: Comparison of commercial wrist-based and smartphone accelerometers, actigraphy, and PSG in a clinical cohort of children and adolescents. J Clin Sleep Med 2016, 12(3):343-350.

22. Lee PH, Tse ACY: Calibration of Wrist-worn ActiWatch 2 and ActiGraph wGT3X for Assessment of Physical Activity in Young Adults. Gait Posture 2019, 68:141-149.

23. Sadeh A, Sharkey KM, Carskadon MA: Activity-based sleep-wake identification: An empirical test of methodological issues. Sleep 1994, 17(3):201-207.

24. Przybylski AK: Digital screen time and pediatric sleep: Evidence from a preregistered cohort study. J Pediatr 2019, 205:218-223.

25. Cabré-Riera A, Torrent M, Donaire-Gonzalez D, Vrijheid M, Cardis E, Guxens M: Telecommunication devices use, screen time and sleep in adolescents. Environ Res 2019, 171:341-347.

26. Fobian AD, Avis K, Schwebel DC: The impact of media use on adolescent sleep efficiency. J Dev Behav Pediatr 2016, 37(1):9-14.

27. Murdock KK, Horissian M, Crichlow-Ball C: Behav Sleep Med. 152016, 3(228-241).

28. Twenge JM, Hisler GC, Krizan Z: Associations between screen time and sleep duration are primarily driven by portable electronic devices: evidence from a population-based study of U.S. children ages 0-17. Sleep Med 2019(article in press).

29. Wood B, Rea MS, Plitnick B, Figueiro MG: Light level and duration of exposure determine the impact of selfluminous tablets on melatonin suppression. App/ Ergon 2013, 44(2):237-240.

30. Wang PY, Chen KL, Yang SY, Lin PH: Relationship of sleep quality, smartphone dependence, and health-related behaviors in female junior college students. PLoS One 2019, 14(4):e0214769.

31. Amra B, Shahsavari A, Shayan-Moghadam R, Mirheli O, Moradi-Khaniabadi B, Bazukar M, Yadollahi-Farsani A, Kelishadi R: The association of sleep and late-night cell phone use among adolescents. J Pediatr (Rio J) 2017, 93(6):560-567.

32. Carter B, Rees P, Hale L, Bhattacharjee D, Paradkar M: Association between portable screen-based media device access or use and sleep outcomes: A systematic review and meta-analysis. JAMA Pediatr 2016, 170(12):12021208.

33. Lemola S, Perkinson-Gloor N, Brand S, Dewald-Kaufmann JF, Grob A: Adolescents' electronic media use at night, sleep disturbance, and depressive symptoms in the smartphone age. J Youth Adolescence 2015, 44:405-418.

34. Mireku MO, Barker MM, Mutz J, Dumontheil I, Thomas MSC, Röösli M, Elliott P, Toledano MB: Night-time screenbased media device use and adolescents' sleep and healthrelated quality of life. Environ Int 2019, 124:66-78.

35. Demirci K, Akgönül M, Akpinar A: Relationship of smartphone use severity with sleep quality, depression, and anxiety in university students. 4 2015, 2(85-92).

36. Dunican IC, Martin DT, Halson SL, Reale RJ, Dawson BT, Caldwell JA, Jones MJ, Eastwood PR: The effects of the removal of electronic devices for 48 hours on sleep in elite judo athletes. J Strength Cond Res 2017, 31(10):28322839.

37. Dworak M, Schierl T, Bruns T, Struder HK: Impact of singular excessive computer game and television exposure on sleep patterns and memory performance of school-aged children. Pediatrics 2007, 120(5):978-985.

38. Woods HC, Scott H: \#Sleepyteens: Social media use in adolescence is associated with poor sleep quality, anxiety, depression and low self-esteem. J Adolescence 2016, 51:41-49. 
39. Nursalam N, Octavia M, Tristiana RD, Efendi F: Association between insomnia and social network site use in Indonesian adolescents. Nurs Forum 2019, 54:149-156.

40. Exelmans L, Van den Bulck J: Bedtime mobile phone use and sleep in adults. Soc Sci Med 2016, 148:93-101.

41. Yland J, Guan S, Emanuele E, Hale L: Interactive vs passive screen time and nighttime sleep duration among school-aged children. Sleep Health 2015, 1:191-196.

42. Kenney EL, Gortmaker SL: United States adolescents' television, computer, videogame, smartphone, and tablet use: Associations with sugary drinks, sleep, physical activity, and obesity. J Pediatr 2017, 182:144-149.

43. Howe KB, Suharlim C, Ueda P, Howe D, Kawachi I, Rimm EB: Gotta catch'em all! Pokémon GO and physical activity among young adults: difference in differences study. BMJ 2016, 355:i6270.

44. Wong FY: Influence of Pokémon Go on physical activity levels of university players: A cross-sectional study. Int $J$ Health Geogr 2017, 16:8.

45. Ernsting C, Dombrowski SU, Oedekoven M, O'Sullivan JL, Kanzler M, Kuhlmey A, Gellert P: Using smartphones and health apps to change and manage health behaviors: A population-based survey. J Med Internet Res 2017, 19(4):e101.

46. Carroll JK, Moorhead A, Bond R, LeBlanc WG, Petrella RJ, Fiscella K: Who uses mobile phone health apps and does use matter? A secondary data analytics approach. J Med Internet Res 2017, 19(4):e125. 